\title{
Distal Transradial Access for Coronary Angiography and Interventions in Everyday Practice: Data From the TRIANGLE Registry (TwitteR Initiated registry for coronary ANgiography in Germany via distaL radial accEss)
}

\author{
Karsten Schenke (D) A Achim Viertel • Nader Joghetaei • \\ Rostislav Prog • Timm Matthiesen · Simon Ohm • Thorsten Dill • \\ Lorenz Bott-Flügel · Gerian Grönefeld
}

Received: February 6, 2021 / Accepted: March 19, 2021 / Published online: April 5, 2021

(c) The Author(s) 2021

\begin{abstract}
Introduction: Transradial access (TRA) has become the primary route for coronary angiography (CAG) and percutaneous coronary interventions (PCI). Recently a new puncture site more distally in the area of the anatomical snuffbox has been described. With this multicenter registry, we wish to demonstrate the feasibility and safety of the distal radial access (dRA).
\end{abstract}

K. Schenke $(\bowtie) \cdot$ A. Viertel · T. Matthiesen ·

G. Grönefeld

Medical Clinic/Cardiology, Asklepios Klinik

Barmbek, Rübenkamp 220, 22291 Hamburg,

Germany

e-mail: k.schenke@asklepios.comA. Viertel

e-mail: a.viertel@asklepios.comT. Matthiesen e-mail:

t.matthiesen@asklepios.comG. Grönefeld

e-mail: g.groenefeld@asklepios.com

N. Joghetaei · L. Bott-Flügel

Klinikum Landkreis Erding, Bajuwarenstraße 5, 85435 Erding, GermanyN. Joghetaei

e-mail: nader.joghetaei@klinikum-erding.de

L. Bott-Flügel

e-mail: lorenz.bott-fluegel@klinikum-

erding.de

R. Prog $\cdot$ S. Ohm · T. Dill

Sana Krankenhaus Benrath, Urdenbacher Allee 83, 40593 Düsseldorf, GermanyR. Prog e-mail: rostislav.prog@Sana.deS. Ohm

e-mail: simon.ohm@Sana.deT. Dill

e-mail: thorsten.dill@Sana.de
Methods: Between December 2018 and May 2019 all patients with a planned CAG or PCI via dRA in three cardiology centers in Germany were entered into this registry. Procedural data, puncture success, crossover rate and complications were registered. Proximal and distal radial artery patency were examined by ultrasound within $48 \mathrm{~h}$.

Results: A total of 327 patients were enrolled (mean age: $69 \pm 12$ years, 69\% male gender, $49 \%$ PCI), in 5 cases bilateral distal puncture was performed. Puncture success, defined as completed sheath placement was high $(N=316 / 332,95 \%)$ and the crossover rate was low $(27 / 332,8 \%)$. The rate of proximal radial artery occlusion after $1-48 \mathrm{~h}$ was low (2/332 $1 \%)$, the rate of occlusion at the distal puncture site was also very low (3/332, 1\%). Major complications were not encountered.

Conclusion: Coronary angiography and interventions via the distal transradial access in the area of the anatomical snuffbox can be performed with a high rate of success and safety. This data suggests a reduced rate of radial artery occlusion compared to previously reported data after cannulation via the standard forearm radial artery puncture site. Randomized studies are needed to further investigate these results.

Trial Registration: This study was registered in the German registry for clinical trials: DRKS00017110, retrospectively on 07.May 2019 
Keywords: Anatomical snuffbox; angiography; Percutaneous interventions; Radial artery occlusion; Transradial access

\section{Key Summary Points}

Why carry out this study?

This is a multicenter trial to investigate the feasibility and safety in performing a coronary angiography or intervention via distal radial access in the area of the anatomical snuffbox

Hypothesis: The use of distal radial access leads to a very low rate of radial artery occlusion and can be performed with a high puncture and procedural success

\section{What was learned from the study?}

In a total number of 327 patients ultrasound examination revealed a very low rate of radial artery occlusion (1\%). We observed a high cannulation success rate (95\%) and a low rate of complications

Coronary angiography and interventions via distal radial access can be performed with a high rate of success and safety. This data underscores the proposed low rate of radial artery occlusion, which might prove to be a benefit compared with conventional proximal access

\section{DIGITAL FEATURES}

This article is published with digital features, including a summary slide, to facilitate understanding of the article. To view digital features for this article go to https://doi.org/10.6084/ m9.figshare.14237840.

\section{INTRODUCTION}

The site of arterial access for coronary angiography and intervention has been the focus of research for decades as it is the source of major complications especially bleeding. In the early 90s, the transradial access (TRA) using a puncture on the forearm over the wrist was developed [7, 14, 32] and, over time, has shown reduced mortality when compared to the transfemoral access (TFA) $[1,6,9,17,28,33,34]$. This data has prompted a Class I and IIa recommendation in current European and American Guidelines $[11,12,20,27]$. The standard puncture site for access to the radial artery is on the lower forearm 2-3 cm proximal to the wrist (proximal radial access, pRA). Dedicated sheath technique, specific guides and closure devices were developed and helped to enable interventional cardiologists in performing nearly any interventional coronary procedure ranging from $4 \mathrm{~F}$ diagnostic angiogram to complex intervention using $6 \mathrm{~F}$ and even $7 \mathrm{~F}$ guides [22, 26].

A typical complication of pRA is a chronic occlusion of the radial artery (RAO) caused by local hematoma, spasm, arterial wall damage or compression either manually or using a dedicated device to allow puncture site closure. The incidence of RAO after pRA in the literature varies between 1 and $10 \%[4,30,36]$. Right now, the standard is patent hemostasis with a minimal duration of compression and the application of heparin [24, 26].

The radial artery runs from the forearm to the base of the thumb and continues into the superficial and deep palmar arch [31]. In the $70 \mathrm{~s}$, the access to the radial artery via the anatomical snuffbox (fovea radialis) has been described as an alternative access point for blood pressure monitoring in children and adults [2]. But it took several decades until the distal radial access (dRA) started to be used for coronary angiogram and intervention $[5,10,13]$.

In 2017 an innovative YouTube (c) Video by one of the inventors of TRA, Dr. Kiemeneij (https://www.youtube.com/watch?v=- 
If5oAF0KJo) and the use of social media (Twitter@) helped to spread the dRA around the globe and sparked several authors to explore this new puncture site $[16,19,21,23,29,37]$. One of the main clinical advantages of dRA that was consistently reported in these articles was a very low rate of RAO.

There has been some debate about the impact of social media on the rapid and worldwide adoption of dRA [35] before enough data could be collected. We designed this registry to systematically examine all patients that were scheduled for distal radial access and further collect data on safety, efficacy and evaluate the $\mathrm{RAO}$ rate.

The distal radial access can be subdivided into two different sites [31], one being the anatomical snuffbox (SB) itself (Fig. 1, blue arrow) and one being distal dorsal (DD) of the tendon of the extensor pollicis longus (Fig. 1, red arrow). Both sites may have different advantages and disadvantages. We documented the exact location of the puncture in this registry to try to elucidate any differences in complications or success rate.
The purpose of the present registry was to retrospectively elucidate potential benefits and risks of this new puncture site compared to the usual proximal radial access site in an all-comer patient population and a multicenter setting.

\section{METHODS}

The TRIANGLE- Registry (TwitteR Initiated registry for coronary ANgiography in Germany via distaL radial accEss) was an observational registry of consecutive patients with acces via dRA. This trial was registered in the German registry for clinical trials: DRKS00017110, retrospectively on 07.May 2019. We anonymously collected the data of all patients in whom a puncture of the radial artery in the area of the snuffbox or distal dorsal via either arm had been attempted between December 2018 and May 2019 in three cardiology centers in Germany in a standardized form. The details of puncture technique has been described elsewhere $[29,31]$. Regarding sheath and catheter material and size, we used identic products for the distal

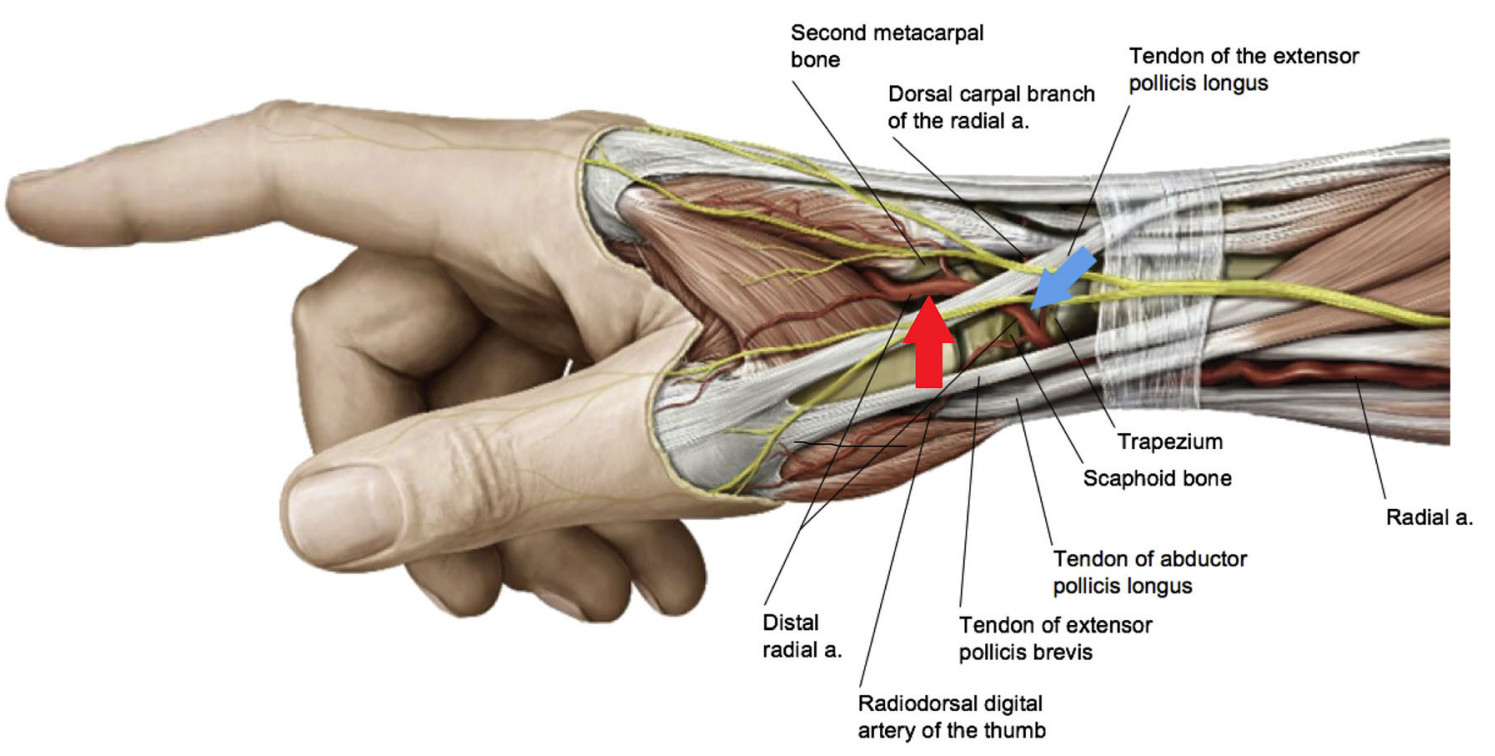

Fig. 1 Anatomy of the radial artery through the anatomical snuffbox. The blue arrow shows the puncture site for snuffbox access, the red arrow marks the site for distal dorsal access. [31] Reproduced with permission from Sgueglia GA, Di Giorgio A, Gaspardone A, Babunashvili A (2018) Anatomic Basis and Physiological Rationale of
Distal Radial Artery Access for Percutaneous Coronary and Endovascular Procedures. JACC Cardiovasc Interv 11:2113-2119 
and proximal access. The sheath used most frequently was "Glidesheath Slender" 6 French (Terumo Corp, Tokyo, Japan). The protocol for spasm prevention and treatment follows uniform principles of mild sedation by means of diazepam and flushing the sheath with 0.2-0.3 mg NTG (Nitroglycerine) i.a. and escalating to morphine iv with prolonged and increasing pain. At the end of the procedure, closure of the puncture site was achieved by a dedicated compression band ("Safeguard", Merrit Co., USA or "TR-Band", Terumo Corp) in all cases following the principle of patent hemostasis described elsewhere [24]. Most frequently, diagnostic and guiding catheters were used with a standard length of $100 \mathrm{~cm}$. In rare cases, where aortic elongation or patient size required longer material, $110 \mathrm{~cm}$ catheters were used ("Launcher", Medtronic Inc., Minneapolis, Minn., USA or "Radiofocus Optitorque", Terumo Corp, Tokyo, Japan).

For intraprocedural anticoagulation, we exclusively used unfractionated heparin (UFH). At the beginning of the angiogram, 2000-5000 units were administered according to internal protocol. In the case of a PCI, heparin was administered either guided by activated clotting time (ACT) 2 centers or by 2500 units every 30 min (1 center). We did not encounter any thrombotic device-associated complications with either regime.

We collected data regarding baseline demographic features, indication, intervention, puncture success rate, security data and RAO. All patients were examined with ultrasound for patency of the vessel within $1-48 \mathrm{~h}$ after removal of the closure device. RAO was defined as loss of antegrade flow assessed by color doppler. Hematoma was stratified into 2 categories $(0-3 \mathrm{~cm},>3 \mathrm{~cm})$. The participating operators each had experience in distal puncture technique over 6 months in more than 50 cases.

To standardize data collection, we defined the access time as "the time between needle in the hand of the operator and the introduction of the sheath", which made our analysis independent from internal cath lab protocols. It allowed us to compare access time and success rate of the different dRA sites (SB and DD) and left and right patient side. Puncture success was defined as successful placement of the sheath. Access site crossover was defined as a procedure that could not be completed using the primary chosen distal access, the selection of an alternative access was left to the discretion of the operator.

This study has been reviewed for all participating institutions by the local IRB of the first author and approval was considered not required due to the retrospective and anonymous data collection. A patient informed consent for participation was deemed not necessary (Ethikkommission Hamburg WF under the number 75-18). All study related procedures were performed in accordance with the Helsinki Declaration. Patient consent was provided for the publication of the images of the patient used.

\section{Statistical Analysis}

For continuous variables the results are displayed as mean \pm standard deviation (SD), they were compared using the Welch Two Sample t-test. For categorical variables the data are given as percentages and counts, they were compared using the chi-square test or Fisher's exact test. The software that was used was $R$ Core Team 2019 (https://www.r-project.org/). A $p$ value $<0.05$ was considered statistically significant.

\section{RESULTS}

\section{Baseline Characteristics}

We included 332 distal punctures including five bilateral distal punctures for CTO (chronic total occlusion) procedures that were performed in 327 patients. Distal transradial access (dRA) was attempted in patients with a palpable pulse without ultrasound screening or guiding. Radial access was achieved using the left (ldRA) or right arm (rdRA). The operator was allowed to decide whether to attempt puncture at snuffbox (SB) or distal-dorsal site (DD) or both. Clinical characteristics are listed in Table 1.102 patients were female (31\%). Our population demonstrated a 
Table 1 Clinical characteristics

\begin{tabular}{ll}
\hline & $N=327$ \\
\hline Age (years) & $69 \pm 12$ \\
Gender: female/male, \% (n) & $31 \%(102) / 69 \%(225)$ \\
Height $(\mathrm{cm})$ & $172 \pm 9$ \\
Weight $(\mathrm{kg})$ & $82 \pm 18$ \\
Body mass index $\left(\mathrm{kg} / \mathrm{m}^{\wedge} 2\right)$ & $27.7 \pm 5.7$ \\
\hline
\end{tabular}

Data expressed as number of non-missing values (\%) $\mathrm{x} \pm \mathrm{s}$ represents mean \pm Standard deviation

mean age of 69 years. $4 \%$ of the patients included had a history of previous coronary artery bypass surgery.

The indication for CAG of the population was an acute coronary syndrome in 49\% (Table 2), including 28 patients with ST elevation myocardial infarction (9\%).

\section{Clinical Outcomes}

Overall PCI rate was $48 \%$ ( $n=158)$. PCI cases did not demonstrate a crossover rate higher than seen with diagnostic angiograms (Table 5). Of note, PCI on chronic total occlusion (CTO) was performed in 16 cases (9.8\% of all PCI cases) including bilateral dRA (Table 3). 10 patients underwent PCI of unprotected left main coronary artery (6\%). LAD (left anterior descending) was the target vessel in 81 cases $(51 \%$ of PCI cases) followed by the right coronary artery (RCA; $n=49,31 \%)$. IVUS (intravascular ultrasound)-and instantaneous wave free

Table 2 Indications for angiography

\begin{tabular}{ll}
\hline & $N=327$ \\
\hline STEMI, \% (n) & $9 \%(28)$ \\
NSTEMI, \% (n) & $33 \%(109)$ \\
stable coronary heart disease, \% (n) & $51 \%(166)$ \\
others, \% (n) & $7 \%(24)$ \\
\hline
\end{tabular}

Data expressed as number of non-missing values (\%) $\mathrm{x} \pm \mathrm{s}$ represents mean \pm Standard deviation
ratio(iFR)/fractional flow reserve (FFR)- guided PCI were performed in 11 (3.3\%) and 17 (5.1\%) cases respectively.

A 6-in-5F slender sheath was used most frequently in this registry $(n=221 / 332,67 \%)$, followed by a long $(23 \mathrm{~cm})$ hydrophilic $6 \mathrm{~F}$ sheath $(n=95 / 332,29 \%)$. We also had a few cases with 7 -in-6F slender sheaths $(n=7,2 \%)$, used in CTOPCI. The majority of the cases were performed through the right radial artery $(n=258 / 332$, $78 \%$ ) (Table 4). SB and DD access were evenly distributed.

The mean access time was 89 seconds, with a small but significantly shorter time for SB access (81 s) compared to DD (99s; $p=0,01$ ). It should be noted, that the mean access time in the 28 patients with ST segment elevation myocardial infarction (STEMI) was only $74 \mathrm{~s}$ without crossover to another access site. The mean duration of the procedure was $41 \mathrm{~min}$ with a mean fluoroscopy time of $9.1 \mathrm{~min}$ and radiation dose of $1967 \mathrm{cGy} \bullet \mathrm{cm} 2$. Mean contrast volume was $88 \mathrm{ml}$ (Table 3 ).

Access site crossover rate was $n=27 / 332$ (8\%) without relevant differences between SB and $\mathrm{DD}(p=0.99)$. Right and left radial access had a similar crossover rate (8\%). Most frequently the operator chose the contralateral radial access $(n=17,63 \%)$. In 10 cases the transfemoral route was used (37\%). The most common reason for crossover was radial loop $(n=8,33 \%)$ followed by spasm. The latter occurred in 4 cases: it was more commonly encountered with SB access (3 cases, $75 \%$ ) compared to DD (1 case $25 \%$ ) (Tables 5, 6).

The fluoroscopy time was numerically longer in DD cases compared to SB (9:56 vs. 8:06 minutes, $p=0.36$ ). We found very similar dose area products and contrast volumes (s. Table 7). The SB access was used slightly more often than DD for PCI-56\% vs. $44 \%$ respectively.

The mean access time was 25 seconds faster in case of rdRA compare to ldRA -84 vs. 109 seconds respectively (Table 6). This difference reached statististical difference $(p=0.001)$. Comparing fluoroscopy data between right and left sided access we found a longer fluoroscopy time via ldRA (10:09 $\mathrm{min})$ and a higher dose area product (2134 cGy $\mathrm{x} \mathrm{\textrm {cm } ^ { 2 }}$ ) compared to rdRA (8:06 $\mathrm{min}, 1907 \mathrm{cGyx} \mathrm{cm}^{2}$ ). The difference 
Table 3 Coronary artery treated

\begin{tabular}{ll}
\hline Coronary artery & Number of PCI $=\mathbf{1 5 8}$ \\
\hline Left main, \% $(n)$ & $6 \%(10)$ \\
RIVA, \% $(n)$ & $51 \%(81)$ \\
RCX (Ramus circumflexus), \% $(n)$ & $23 \%(37)$ \\
RCA, \% (n) & $31 \%(49)$ \\
Bypass vein graft, \% $(n)$ & $1 \%(4)$ \\
\hline
\end{tabular}

Data expressed as number of non-missing values (\%)

Table 4 Procedural characteristics

\begin{tabular}{cc}
\hline Type of intervention $(\boldsymbol{N}=327)$ & \\
\hline PCI, \% $(n)$ & $48 \%$ \\
IVUS, \% $(n)$ & $3 \%(158)$ \\
iFR/FFR, \% $(n)$ & $5 \%(17)$ \\
Multivessel, \% of PCI $(n)$ & $9 \%(14)$ \\
CTO, \% of PCI $(n)$ & $11 \%(18)$ \\
Sheath size $(N=332)$ & \\
4F, \% $(n)$ & $3 \%(9)$ \\
6-in-5Fslender, \% (n) & $67 \%$ \\
& $(221)$ \\
7-in-6Fslender, $\%(n)$ & $2 \%(7)$ \\
6F, \% $(n)$ & $29 \%(95)$ \\
\hline
\end{tabular}

Data expressed as number of non-missing values (\%)

in fluoro time was statistically significant $(p=$ 0.017).

Ultrasound-guided patency of the radial artery was demonstrated within 48 hours after removal of the closure device, with 14 cases being lost to follow-up (4\%). We observed three occlusions of the RA at the distal puncture site and in two of these cases also an occlusion on the forearm.

Access-related hematoma occurred in 17 cases $(5.1 \%)$. Of all the SB punctures $(n=180)$, there were 8 cases with access site-related hematoma (5\%). Regarding DD puncture $(n=152)$, hematoma rate was similar $(5.9 \%)$. All of these hematomas were mild (maximal area 3 $\times 3 \mathrm{~cm}$ ), managed conservatively, and without need for transfusion or surgical intervention.

A total of 57 patients had a body height of more than $180 \mathrm{~cm}$, in almost all of these cases the standard catheter length was sufficient to complete the procedure. Only in 5 cases distal access necessitated the use of longer catheters $(110 \mathrm{~cm})(1.5 \%)$. Of these 5 cases rRA (right radial artery) was the selected site in 4 cases (80\%). The mean height of these patients was $188 \mathrm{~cm}$, whereas the entire cohort had a mean height of $172 \mathrm{~cm}$.

\section{DISCUSSION}

The results of this patient registry undergoing coronary angiography or intervention using the distal radial access underscores the feasibility and safety of this new technique. In 327 patients with 332 punctures the planned procedure could be completed using the dRA in $92 \%(305 / 332)$ even in complex cases, such as STEMI, Left main (LM) -PCI and CTO-PCI.

These results demonstrating a very high success rate and very low rate of RAO are in line with previously published registries with dRA. Dr Kiemeneij [13] has introduced ldRA as the primary access route for right-handed patients. In this article, he reported that the puncture success rate was $89 \%$ with 8 failed attempts in a total of 70 consecutive patients. In this registry, there was one case of distal RAO and no case of RAO at the forearm site. Lee et al.[19] published 
Table 5 Procedural endpoints

\begin{tabular}{llll}
\hline & Total $\boldsymbol{N}=\mathbf{3 3 2}$ & CAG $\boldsymbol{N}=\mathbf{1 6 6}$ & PCI ink1. IVUS/IFR/FFR $\boldsymbol{N}=\mathbf{1 6 6}$ \\
\hline Puncture success, \% (n) & $95 \%(316)$ & $95 \%(158)$ & $95 \%(158)$ \\
Crossover, \% $(n)$ & $8 \%(27)$ & $7 \%(11)$ & $10 \%(16)$ \\
Access: DD, \% $(n)$ & $46 \%(152)$ & $48 \%(79)$ & $44 \%(73)$ \\
Access: SB, \% $(n)$ & $54 \%(180)$ & $52 \%(87)$ & $56 \%(93)$ \\
Duration of the procedure; min & $40 \pm 32$ & $21 \pm 18$ & $59 \pm 32$ \\
Contrast, ml & $88 \pm 64$ & $45 \pm 28$ & $129 \pm 63$ \\
DAP (Dose area product) cGy x cm & $1955 \pm 1995$ & $1069 \pm 944$ & $2815 \pm 2343$ \\
\hline
\end{tabular}

Data expressed as number of non-missing values (\%) $\mathrm{x} \pm \mathrm{s}$ represents mean \pm Standard deviation

Table 6 Left versus right radial

\begin{tabular}{lllll}
\hline & $\begin{array}{l}\text { Total } \\
\boldsymbol{N}=\mathbf{3 3 2}\end{array}$ & $\begin{array}{l}\text { Right radial } \\
\boldsymbol{N}=\mathbf{2 5 8}\end{array}$ & $\begin{array}{l}\text { Left radial } \\
\boldsymbol{N}=\mathbf{7 4}\end{array}$ & $\begin{array}{l}\boldsymbol{p} \text { - value left vs. } \\
\text { right }\end{array}$ \\
\hline Access time $(\mathrm{sec})$ & $89 \pm 79$ & $84 \pm 76$ & $109 \pm 88$ & 0.002 \\
Puncture success, \% $(n)$ & $95 \%(316)$ & $95 \%(245)$ & $96 \%(71)$ & 0.99 \\
Crossover, \% $(n)$ & $8 \%(27)$ & $8 \%(21)$ & $8 \%(6)$ & 0.99 \\
Fluoro time (min) & $9.2 \pm 9.8$ & $8.7 \pm 9.3$ & $11.3 \pm 11.4$ & 0.017 \\
DAP (cGY x cm $\left.{ }^{2}\right)$ & $1955 \pm 1995$ & $1907 \pm 2016$ & $2134 \pm 1923$ & 0.117 \\
Contrast (ml) & $88 \pm 64$ & $88 \pm 66$ & $87 \pm 59$ & 0.66 \\
Forearm radial artery occlusion, \% & $1 \%(2)$ & $1 \%(2)$ & $0(0)$ & n.a \\
$\quad(n)$ & & & & \\
Distal radial artery occlusion, \% $(n)$ & $1 \%(3)$ & $1 \%(3)$ & $0(0)$ & n.a \\
Any hematoma, \% $(n)$ & $5 \%(17)$ & $6 \%(15)$ & $3 \%(2)$ & 0.38 \\
\hline
\end{tabular}

Data expressed as number of non-missing values (\%) $\mathrm{x} \pm \mathrm{s}$ represents mean \pm Standard deviation

data describing 200 consecutive patients in a single center using ldRA as a primary access. The observed success rate of arterial puncture was $95.5 \%(191 / 200)$ which was nearly identical to our rate of $95 \%$. The patency of the distal radial artery was examined by ultrasound in 141 cases, no dRAO was diagnosed. $7.4 \%$ of the patients had minor hematoma, no major hematoma was found.

The success rate of sheath placement was found to be $95 \%$ (316/332), without differences between side (ldRA or rdRA) or site (SB or DD) of the dRA. The crossover rate was $8 \%$, mainly due to radial looping and/or radial spasm. These results are comparable to data from other registries. In a review article on 1118 patients, the observed cannulation success rate was between 88 and 100\% [21]. In our opinion, the problems that were encountered in our registry would have forced a crossover after pTRA in the same patients. However, we must admit that an adequately powered randomized trial is necessary to confirm this assumption. From an anatomic point of view, once the guide has crossed the 
Table 7 Access Snuff-box versus dorsal-distal

\begin{tabular}{|c|c|c|c|}
\hline & SB $N=180$ & DD $N=152$ & $p$-value SB vs DD \\
\hline Access time (sec) & $81 \pm 74$ & $99 \pm 84$ & 0.01 \\
\hline Puncture success, $\%(n)$ & $96 \%(172)$ & $95 \%(144)$ & 0.8 \\
\hline Crossover, \% (n) & $7 \%(13)$ & $9 \%(14)$ & 0.65 \\
\hline Fluoro time (min) & $8.7 \pm 9.4$ & $9.9 \pm 10.3$ & 0.36 \\
\hline DAP (cGy x cm²) & $1928 \pm 1713$ & $1987 \pm 2289$ & 0.98 \\
\hline Contrast (ml) & $89 \pm 68$ & $86 \pm 60$ & 0.91 \\
\hline Forearm radial artery occlusion, $\%(n)$ & $1 \%(1)$ & $1 \%(1)$ & n.a \\
\hline Distal radial artery occlusion, \% (n) & $1 \%(2)$ & $1 \%(1)$ & n.a \\
\hline Any hematoma, \% (n) & $4 \%(8)$ & $6 \%(9)$ & 0.7 \\
\hline
\end{tabular}

Data expressed as number of non-missing values $(\%) \mathrm{x} \pm \mathrm{s}$ represents mean \pm Standard deviation

middle forearm RA the route it takes is identical to the pRA access including any upcoming anatomical challenges.

Oliveira et al. report a rate of successful sheath insertion of $98.6 \%$ in a prospective large all-comers registry in 435 Patients [23]. They did not use ultrasound guidance and experienced a very low access site crossover rate of 3\% (13 cases).

The distance between pRA and dRA is approximately 3-4 centimetres (SB) or 5-7 centimetres (DD). Consequently, under certain circumstances (such as arterial tortuosity or looping, Aorta ascendens dilatation etc.) the standard $100 \mathrm{~cm}$ catheter length may not be sufficient for patients taller than $185 \mathrm{~cm}$. In this registry, we included 30 such patients. In five cases, the use of $110 \mathrm{~cm}$ diagnostic and guiding catheters was necessary and allowed completion of the procedure. We recommend a small stock of catheters with a length of $110 \mathrm{~cm}$ to allow distal radial interventions in this cohort. The tallest patient in this registry with a height of over $200 \mathrm{~cm}$ was successfully treated using dRA with a $110 \mathrm{~cm}$ guide (Fig. 2).

To date no data has been published comparing the different anatomical dRA puncture sites: the anatomical snuffbox and distal dorsal site. In our registry, the DD access time was significantly longer than the $\mathrm{SB}$, which could be explained by the smaller size of the radial artery. The puncture success rate and crossover rates were similar after SB and DD punctures. The fluoroscopy time was longer in DD cases compared to SB. However, we found the same dose area products and contrast volumes (s. Table 4).

The size of the distal RA (dRA) compared to the forearm RA is about $20 \%$ smaller $[8,15]$, so inherently the puncture of the dRA is more challenging and in a certain group of patients (esp. smaller women) even impossible. In this registry we did not collect a screening failure log to calculate the rate of patients in which dRA was deemed feasible. In a prospective registry in Brazil 70 consecutive patients were screened by palpation of dRA pulse[3], $61(87 \%)$ of these patients were considered suitable for dRA. Dr. Kiemeneij reports of a rate of 70 patients out of a group of 118 screened patients leading to a rate of $60 \%$ [13] after adjustment for logistical or other reasons the rate would be $70 \%$.

We observed a significantly shorter puncture time in right sided access. We assume that since all participating cath labs use the right side of the patient as standard access, the puncture is more routine for the operator, explaining the difference. Comparing fluoroscopy data between right and left sided access, we found a longer fluoroscopy time and contrast use via ldRA compared to rdRA. These differences could 

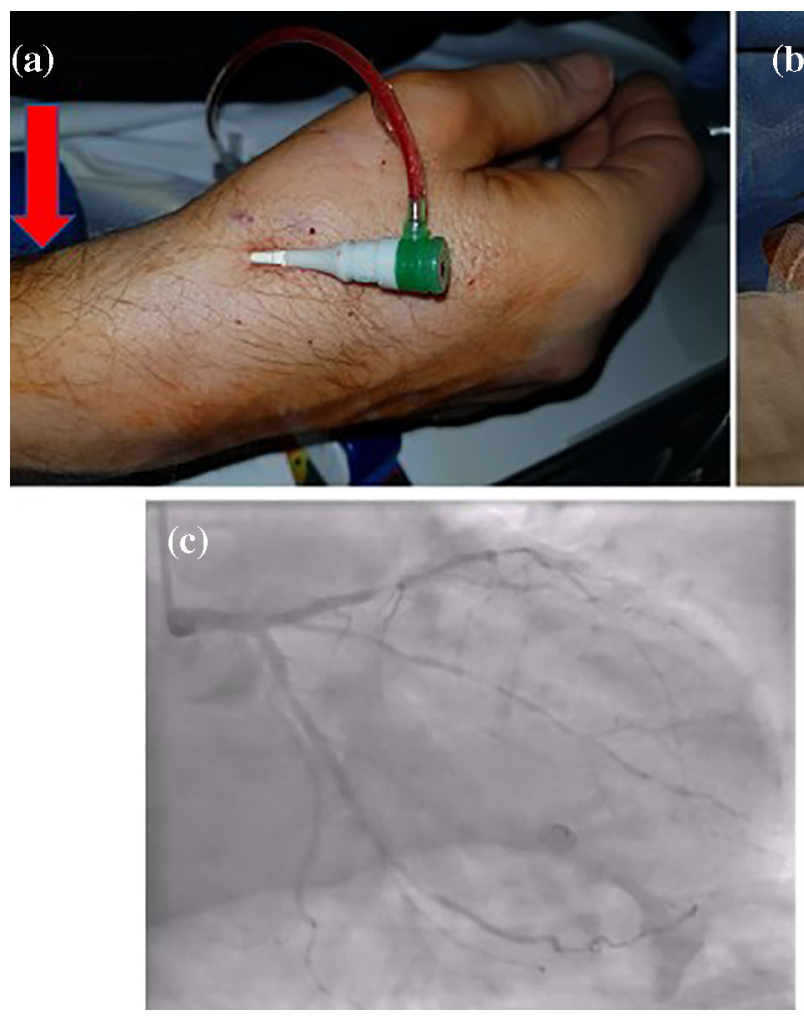

Fig. 2 Patient example, male, 61 years old, $203 \mathrm{~cm}$, $107 \mathrm{~kg}$. rSB access with Glide Sheath Slender 6in5F (Terumo $\left.{ }^{\circledR}\right)$, red arrow shows the puncture site by $p R A$;

be explained by the use of single catheter technique in case of rdRA by some operators and that all post CABG (coronary artery bypass graft) patients were examined using ldRA. In a large randomized trial comparing left-radial, right-radial and femoral access in a 1:1:1 fashion in 1493 patients undergoing cardiac catheterization no significant difference in radiation doses were found, although the operators radiation dose was significantly higher in left radial access [25].

One of the major advantages of the dRA over pRA is the previously reported low rate of forearm radial artery occlusion. The majority of patients in this registry $(318 / 332)$ were followed up by ultrasound assessment of the patency of the RA within $48 \mathrm{~h}$ after the procedure. A radial artery occlusion at the forearm site was found only in 2 cases. We registered 3 distal RAOs. The observed rate of RAO after dRA is lower than the reported rate of $1-10 \%$ in trials and registries on
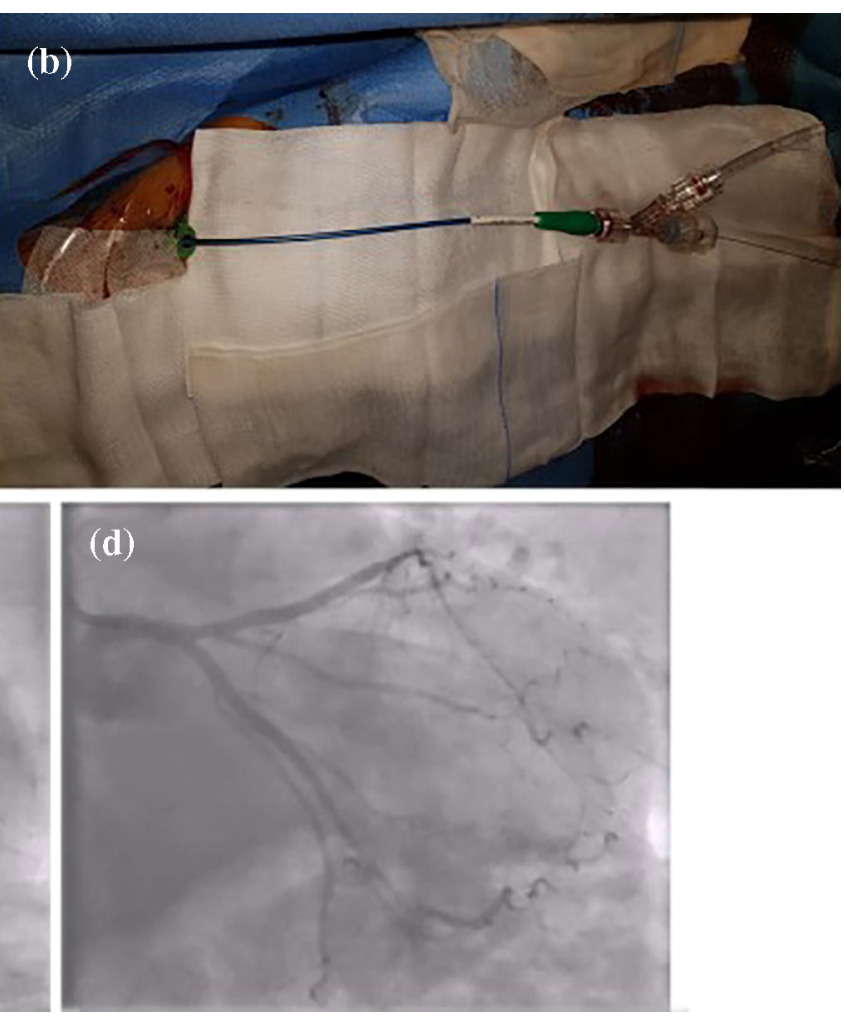

(b) guiding catheter EBU3,5 $\mathrm{SH} 110 \mathrm{~cm}$ (Launcher, Medtronic $\left.{ }^{\circledR}\right)$; LAD and RCX before (c) and after (d) PCI

traditional pRA $[4,18]$. This difference can be explained by the lack of trauma of the wall and preservation of the flow in the radial artery on the forearm through the deep palmar arch.

In 17 cases we found local hematomas ranging from 1 to $3 \mathrm{~cm}$ at the access site, there was no difference between SB and DD site. The same result was found in the four largest of them, up to $3 \mathrm{~cm}$, with equal distribution of 2 hematomas in each group. It is important to mention that 14 of 17 local hematomas were after complex PCIs incl. CTO-PCI, which needed higher doses of heparin. The muscle basement provides enough support during patent hemostasis after the DD puncture while the scaphoid bone provides excellent anchor to compress the RA after SB access.

Of methodological interest, this large allcomer registry is the first social media initiated medical trial. The investigators became acquainted with one another using Twitter ${ }^{\circledR}$ 
during discussions about distal radial access that were started by F. Kiemeneij. At the suggestion of Dr. N. Joghetaei the authors decided to start this multicenter registry. Study protocol, discussion of standards and endpoints, all have been agreed upon via electronic communication only.

\section{LIMITATIONS}

This registry included neither a randomization nor a control group and although we used uniformly prespecified endpoints for the analysis it was a retrospective data collection. It is therefore possible that some patients with complications or crossover may have been overlooked. We tried to counter this problem by double rechecking all cath lab protocols in the participating centers during the period of data acquisition. The data of the patients in the registry were checked for consistency by the interventionalists after completion of the data base. The lack of a control group with proximal access certainly limits our conclusions, we therefore discussed a comparison of our data with prepublished data of pRA.

Furthermore, 14 cases (4\%) were lost to ultrasound follow-up which could have changed the outcome of artery occlusion. However, with $96 \%$ of completed sonographic follow-up in an all-comers' population, our registry is the first one with the widest range of performed PCIs, including STEMI, left main and CTO.

The punctures of the radial artery and the coronary procedures in this registry were performed by experienced interventional cardiologists with mostly more than 10 years of experience, which might have influenced the success and crossover rate compared to institutions with less experience in radial access. The availability and frequent use of dedicated radial access techniques such as a single diagnostic catheter (e.g. TIG II Terumo ${ }^{\circledR}$ ) for both ostia and slender sheaths, as well as pigtail- and ballon assisted tracking in case of spasm could have influenced the success and complication rate.

According to our methods described above, only patients with a palpable pulse were considered for inclusion into the protocol.
Therefore, puncture success in unselected patients might be lower than the reported rate of $95 \%$ in our study.

\section{CONCLUSIONS}

In summary, this register is currently one of the largest truly all-comers studies demonstrating the feasibility of the dRA in both SB and DD punctures sites in various forms of emergent and complex coronary procedures whilst being independent from patient height.

Distal radial access for coronary angiography and interventions is at least a viable alternative to the standard forearm puncture site for every kind of coronary procedure. With this social media inspired registry we demonstrate a high success rate and comparing our findings with historic data we observe a very low risk of access site radial artery occlusion. To further validate these findings and potential benefits for the patients, the results of ongoing randomized controlled trials have to be awaited.

In our opinion, social media opens new possibilities in medical science, for example in organization and conduction of trials. Our registry is the first Twitter-initiated study worldwide that we are aware of.

\section{ACKNOWLEDGEMENTS}

The authors would like to thank Dr. Barry Harrington for his valuable assistance in cross checking the orthography as a native English speaker and Dr. Peter Wohlmuth from Asklepios Proresearch for statistical assistance. Both colleagues have not received monetary compensation for their help. We also thank the staff of all of the participating cath labs in helping us to establish the distal radial access in everyday practice. Last but not least we express our gratitude to all the participants of the study.

Funding. No funding or sponsorship was received for this study or publication of this article. The Rapid Service Fee was funded by the authors. 
Authorship. All named authors meet the International Committee of Medical Journal Editors (ICMJE) criteria for authorship for this article, take responsibility for the integrity of the work as a whole, and have given their approval for this version to be published.

Authors Contributions. Karsten Schenke: Conceptualization, Writing- Original draft preparation, Investigation, Funding acquisition. Achim Viertel: Investigation, Supervision, Validation. Nader Joghetaei: Conceptualization, Investigation. Rostyslav Prog: Conceptualization, Investigation. Timm Matthiesen: Investigation, Resources; Simon Ohm: Investigation, resources; Thorsten Dill: Investigation, Supervision, Lorenz Bott-Flügel: Investigation, Supervision, Gerian Grönefeld: Writing Review and editing, Project administration, Funding Acquisition.

Prior Presentation. A part of this data has been presented as an abstract at the online ESC Congress in November 2020.

Disclosures. Karsten Schenke, Achim Viertel, Nader Joghetaei, Rostyslav Prog, Timm Matthiesen, Simon Ohm, Thorsten Dill, Lorenz Bott-Flügel, Gerian Grönefeld have no conflict of interest to disclose.

Compliance with Ethics Guidelines. This study has been reviewed by the local IRB (Ethikkommission Hamburg under the number WF 75-18). All study related procedures were performed in accordance with the Helsinki Declaration. The authors report that patient consent was provided for the publication of the images of the patient used. The authors report that permission from the copyright owner(s) for both the print and online format was provided for the publication of the image used.

Data Availability. The authors report that the data collected is available for cross checking. This trial was registered in the German registry for clinical trials: DRKS00017110, retrospectively on 07.05.2019.
Open Access. This article is licensed under a Creative Commons Attribution-NonCommercial 4.0 International License, which permits any non-commercial use, sharing, adaptation, distribution and reproduction in any medium or format, as long as you give appropriate credit to the original author(s) and the source, provide a link to the Creative Commons licence, and indicate if changes were made. The images or other third party material in this article are included in the article's Creative Commons licence, unless indicated otherwise in a credit line to the material. If material is not included in the article's Creative Commons licence and your intended use is not permitted by statutory regulation or exceeds the permitted use, you will need to obtain permission directly from the copyright holder. To view a copy of this licence, visit http://creativecommons.org/licenses/by$\mathrm{nc} / 4.0 /$.

\section{REFERENCES}

1. Agostoni P, Biondi-Zoccai GGL, De Benedictis ML, et al. Radial versus femoral approach for percutaneous coronary diagnostic and interventional procedures: Systematic overview and meta-analysis of randomized trials. J Am Coll Cardiol. 2004;44: 349-56. https://doi.org/10.1016/j.jacc.2004.04.034.

2. Amato JJ, Solod E, Cleveland RJ. A ,,second“ radial artery for monitoring the perioperative pediatric cardiac patient. J Pediatr Surg. 1977;12:715-7. https://doi.org/10.1016/0022-3468(77)90399-2.

3. Andrade $\mathrm{P}$, Tebet $\mathrm{M}$, Rinaldi $\mathrm{F}$, et al. Initial experience with left distal transradial access for invasive coronary procedures. J Transcatheter Interv. 2018;16:1-5. https://doi.org/10.31160/ jotci2018;26(1)a0002.

4. Avdikos G, Karatasakis A, Tsoumeleas A, et al. Radial artery occlusion after transradial coronary catheterization. Cardiovasc Diagn Ther. 2017;7: 305-16. https://doi.org/10.21037/cdt.2017.03.14.

5. Babunashvili A, Dundua D. Recanalization and reuse of early occluded radial artery within 6 days after previous transradial diagnostic procedure. Catheter Cardiovasc Interv. 2011;77:530-6. https:// doi.org/10.1002/ccd.22846.

6. Bernat I, Horak D, Stasek J, et al. ST-segment elevation myocardial infarction treated by radial or 
femoral approach in a multicenter randomized clinical trial: The STEMI-RADIAL trial. J Am Coll Cardiol. 2014;63:964-72. https://doi.org/10.1016/j. jacc.2013.08.1651.

7. Campeau L. Percutaneous radial artery approach for coronary angiography. Cathet Cardiovasc Diagn. 1989;16:3-7.

8. Davies RE, Gilchrist IC. Back hand approach to radial access: The snuff box approach. Cardiovasc Revascularization Med. 2018;19:324-6. https://doi. org/10.1016/j.carrev.2017.08.014.

9. Jolly SS, Yusuf S, Cairns J, et al. Radial versus femoral access for coronary angiography and intervention in patients with acute coronary syndromes (RIVAL): a randomised, parallel group, multicentre trial. Lancet. 2011;377:1409-20. https://doi.org/10.1016/S0140-6736(11)60404-2.

10. Kaledin AL, Kochanov IN, Seletskiı̌ SS, et al. Peculiarities of arterial access in endovascular surgery in elderly patients. Adv Gerontol. 2014;27:115-9.

11. Kastrati A, Banning AP, Koller A, et al. 2018 ESC/ EACTS Guidelines on myocardial revascularization. Eur Heart J. 2018;40:87-165. https://doi.org/10. 1093/eurheartj/ehy394.

12. Kastrati A, Caforio ALP, Bucciarelli-Ducci C, et al. 2017 ESC Guidelines for the management of acute myocardial infarction in patients presenting with ST-segment elevation: the task force for the management of acute myocardial infarction in patients presenting with ST-segment elevation of the European Society of Cardiology (ESC). Eur Heart J. 2017;39:119-77. https://doi.org/10.1093/eurheartj/ ehx393.

13. Kiemeneij F. Left distal transradial access in the anatomical snuffbox for coronary angiography (ldTRA) and interventions (ldTRI). EuroIntervention. 2017;13:851-7. https://doi.org/10.4244/EIJ-D17-00079.

14. Kiemeneij F, Jan Laarman G. Percutaneous transradial artery approach for coronary stent implantation. Cathet Cardiovasc Diagn. 1993;30:173-8. https://doi.org/10.1002/ccd.1810300220.

15. Kim MC, Kim JH, Ahn Y, et al. Gender differences in the distal radial artery diameter for the snuffbox approach. Cardiol J. 2018;25:639-41. https://doi. org/10.5603/cj.2018.0128.

16. Kim Y, Ahn Y, Kim I, et al. Feasibility of coronary angiography and percutaneous coronary intervention via left snuffbox approach. Korean Circ J. 2018;48:1120-30. https://doi.org/10.4070/kcj. 2018.0181 .
17. Kolkailah AA, Alreshq RS, Muhammed AM et al (2018) Transradial versus transfemoral approach for diagnostic coronary angiography and percutaneous coronary intervention in people with coronary artery disease. Cochrane database Syst Rev 4: CD012318. doi: https://doi.org/10.1002/14651858. CD012318.pub2

18. Kotowycz MA, Džavík V. Radial artery patency after transradial catheterization. Circ Cardiovasc Interv. 2012;5:127-33. https://doi.org/10.1161/CIRCINT ERVENTIONS.111.965871.

19. Lee J-W, Park SW, Son J-W, et al. Real-world experience of the left distal transradial approach for coronary angiography and percutaneous coronary intervention: a prospective observational study (LeDRA). EuroIntervention. 2018;14:e995-1003. https://doi.org/10.4244/EIJ-D-18-00635.

20. Levine GN, Bates ER, Blankenship JC, et al. 2011 ACCF/AHA/SCAI guideline for percutaneous coronary intervention. J Am Coll Cardiol. 2011;58: e44-122. https://doi.org/10.1016/j.jacc.2011.08. 007.

21. Liontou C, Kontopodis E, Oikonomidis N, et al. Distal radial access: a review article. Cardiovasc Revascularization Med. 2020;21:412-6. https://doi. org/10.1016/j.carrev.2019.06.003.

22. Mason PJ, Shah B, Tamis-Holland JE, et al. An update on radial artery access and best practices for transradial coronary angiography and intervention in acute coronary syndrome: a scientific statement from the American Heart Association. Circ Cardiovasc Interv. 2018;11:1-21. https://doi.org/10.1161/ HCV.0000000000000035.

23. Oliveira MDP, Navarro EC, Kiemeneij F. Distal transradial access as default approach for coronary angiography and interventions. Cardiovasc Diagn Ther. 2019;9:513-9. https://doi.org/10.21037/cdt. 2019.09.06.

24. Pancholy S, Coppola J, Patel T, Roke-Thomas M. Prevention of radial artery occlusion-patent hemostasis evaluation trial (PROPHET study): a randomized comparison of traditional versus patency documented hemostasis after transradial catheterization. Catheter Cardiovasc Interv. 2008;72:335-40. https://doi.org/10.1002/ccd. 21639.

25. Pancholy SB, Joshi P, Shah S, et al. Effect of vascular access site choice on radiation exposure during coronary angiography: the REVERE Trial (Randomized Evaluation of Vascular Entry Site and Radiation Exposure). JACC Cardiovasc Interv. 2015;8: 1189-96. https://doi.org/10.1016/j.jcin.2015.03. 026. 
26. Rao SV, Tremmel JA, Gilchrist IC, et al. Best practices for transradial angiography and intervention: a consensus statement from the society for cardiovascular angiography and intervention's transradial working group. Catheter Cardiovasc Interv. 2014;83:228-36. https://doi.org/10.1002/ccd. 25209.

27. Roffi M, Patrono C, Collet J-P, et al. 2015 ESC Guidelines for the management of acute coronary syndromes in patients presenting without persistent ST-segment elevation: task force for the management of acute coronary syndromes in Patients presenting without persistent ST-segment elevation of the European society of cardiology (ESC). Eur Heart J. 2016;37:267-315. https://doi.org/10.1093/ eurheartj/ehv320.

28. Romagnoli E, Biondi-Zoccai G, Sciahbasi A, et al. Radial versus femoral randomized investigation in st-segment elevation acute coronary syndrome: the rifle-steacs (radial versus femoral randomized investigation in st-elevation acute coronary syndrome) study. J Am Coll Cardiol. 2012;60:2481-9. https://doi.org/10.1016/j.jacc.2012.06.017.

29. Schenke K, Prog R, Matthiesen T, et al. Initial experiences with access via the distal radial artery in the anatomical snuffbox for coronary angiography and interventions: Data from the distal radial access for coronary anGiOgraphy and iNtervention (DRAGON) registry. Kardiologe. 2020;14:217-25. https://doi.org/10.1007/s12181-019-00361-4.

30. Sciahbasi A, Rigattieri S, Sarandrea A, et al. Radial artery occlusion and hand strength after percutaneous coronary procedures: results of the HANGAR study. Catheter Cardiovasc Interv. 2016;87:868-74. https://doi.org/10.1002/ccd.26142.

31. Sgueglia GA, Di Giorgio A, Gaspardone A, Babunashvili A. Anatomic basis and physiological rationale of distal radial artery access for percutaneous coronary and endovascular procedures. JACC Cardiovasc Interv. 2018;11:2113-9. https://doi.org/10. 1016/j.jcin.2018.04.045.

32. Stella PR, Kiemeneij F, Laarman GJ, et al. Incidence and outcome of radial artery occlusion following transradial artery coronary angioplasty. Cathet Cardiovasc Diagn. 1997;40:156-8. https://doi.org/ 10.1002/(SICI)1097-0304(199702)40:2\%3c156:: AID-CCD7\%3e3.0.CO;2-A.

33. Valgimigli M, Frigoli E, Leonardi S, et al. Radial versus femoral access and bivalirudin versus unfractionated heparin in invasively managed patients with acute coronary syndrome (MATRIX): final 1-year results of a multicentre, randomised controlled trial. Lancet. 2018;392:835-48. https:// doi.org/10.1016/S0140-6736(18)31714-8.

34. Valgimigli M, Gagnor A, Calabró P, et al. Radial versus femoral access in patients with acute coronary syndromes undergoing invasive management: a randomised multicentre trial. Lancet. 2015;385: 2465-76. https://doi.org/10.1016/S01406736(15)60292-6.

35. Vidovich MI. Twitter, folklore and evidence-based medicine: the tale of distal radial access. Cardiovasc Revascularization Med. 2019;20:638-9. https://doi. org/10.1016/j.carrev.2019.06.004.

36. Zankl AR, Andrassy M, Volz C, et al. Radial artery thrombosis following transradial coronary angiography: incidence and rationale for treatment of symptomatic patients with low-molecular-weight heparins. Clin Res Cardiol. 2010;99:841-7. https:// doi.org/10.1007/s00392-010-0197-8.

37. Ziakas A, Koutouzis M, Didagelos M, et al. Right arm distal transradial (snuffbox) access for coronary catheterization: Initial experience. Hell J Cardiol. 2018. https://doi.org/10.1016/j.hjc.2018.10.008. 